\title{
Uso del Trigger Tool para la Detección de Incidentes y Eventos Adversos en una Mutua Colaboradora con la Seguridad Social
}

\author{
Using Trigger Tool to detect incidents and adverse \\ events in a mutual insurance company that \\ collaborate with the Social Security system
}

\author{
Jordi Ortner Sancho ${ }^{1}$ \\ Rafael Manzanera López ${ }^{1}$ \\ Norma Grau Balcells ${ }^{1}$ \\ Diego José Moya Alcocer ${ }^{1}$ \\ Xavier Farrús Esteban ${ }^{1}$ \\ Jose Miguel Martínez Martínez $z^{1,2,3}$ \\ ${ }^{1}$ MC Mutual, Barcelona, España. \\ ${ }^{2}$ Departamento de Estadística e Investigación Operativa. Universitat Politècnica de Catalunya, Barce- \\ Iona, España. \\ ${ }^{3}$ Grupo de Investigación en Salud Pública. Universidad de Alicante, Alicante, España.
}

Fechas · Dates

Recibido: 2019.07 .26

Aceptado: 2020.04 .15

Publicado: 2020.07.15
Correspondencia · Corresponding Author

Jordi Ortner Sancho

MC Mutual, Departamento Gestión de Asistencia Sanitaria e ITCP. División Servicios Médicos y Asistenciales, Barcelona, España.

jortner@mc-mutual.com 


\section{Resumen}

Objetivo. Evaluar diferencias entre la detección de incidentes o eventos adversos (I/EA) en una mutua laboral, mediante una herramienta tipo Trigger Tool (TT) y una plataforma de notificación voluntaria (SNEA).

Métodos. La población de estudio es la población trabajadora atendida ambulatoriamente en una mutua laboral de Enero a Septiembre del 2016. Se seleccionaron los casos declarados como I/EA según si el evento no ha afectado al paciente o por el contrario le ha afectado, a través del SNEA ( $\mathrm{n}=21$ casos). Por otro lado, se seleccionaron aleatoriamente 20 historias clínicas por mes donde se aplicó la herramienta TT (180 casos). Se adaptaron 11 triggers para detectar I/EA. Se revisaron las 201 historias clínicas buscando la existencia de triggers. Se obtuvo la concordancia entre el sistema SNEA y el TT utilizando la proporción de concordancia positiva (I/EA), proporción de concordancia negativa (no I/EA) e índice Kappa.

Resultados. TT detectó casos de I/EA en el 41,3\% de las revisiones mientras que el SNEA $10,3 \%(p<0,001)$. El índice Kappa ofreció un valor de concordancia baja $(K a p p a=0,12)$ lo que denota la pequeña coincidencia de sucesos adversos detectados por ambos sistemas. La proporción de concordancia negativa fue mayor que la de concordancia positiva $(74,5 \%$ frente a un 26,9\%). El sistema SNEA detectó menos I/EA y sobretodo se trata de menos incidentes. Por el contrario, el sistema TT detectó mayor número de I/EA y especialmente EA.

Conclusiones. Trigger Tool es una herramienta recomendable para la detección de incidentes o eventos adversos que puede complementar la obtenida mediante una plataforma de notificación voluntaria en la realidad de una Mutua laboral.

Palabras clave: Evento adverso; Seguridad del paciente; Trigger Tool

\section{Abstract}

Objective. To evaluate differences between the detection of incidents or adverse events (I/ AE) using a Trigger Tool (TT) and voluntary notification platform (SNEA).

Methods. The study population is the working population attended on an outpatient basis in an Insurance Company ("mutua") from January to September 2016. The cases declared as Incident or Adverse Event ( / AE) were selected through the SNEA (21 cases), according to whether the event has not affected the patient or on the contrary has affected him. On the other hand, 20 clinical histories per month were randomly selected where the TT was applied (180 cases). The 201 clinical histories were reviewed looking for the existence of triggers. The agreement between the SNEA system and the TT was evaluated using proportion of positive agreement (I/EA), proportion of negative agreement (not I/EA) and Kappa index.

Results. TT detected I/EA cases in $41.3 \%$ of the revisions while the SNEA was $10.3 \%$ (p $<0.001)$. The Kappa index showed a low concordance value (Kappa = 0.12), which indicates the small coincidence of I/EA detected by both systems. The proportion of negative agreement was greater than that of positive agreement (74.5\% versus $26.9 \%$ ). The SNEA system detected less I/ EA and above all it deals with fewer incidents. On the contrary, the TT system detected a greater number of I EA and especially EA. 
Conclusions. Trigger Tool is a recommended tool for the detection of incidents or adverse events that can complement the one obtained through voluntary notification platform in the reality of a "mutua".

Palabras clave: Adverse event, Patient safety, Trigger Tool

\section{Introducción}

La seguridad del paciente se entiende como la prevención de eventos adversos (EA) para el paciente derivados de la actividad sanitaria. La seguridad del paciente ha pasado de ser una dimensión más de la calidad asistencial a una forma de entender la atención sanitaria sin la cual ésta carecería de sentido. Esta noción ha experimentado un crecimiento extraordinario en los últimos 20 años impulsado sobre todo por el informe "To Err is Human"(1), elaborado a finales del siglo pasado por el Instituto de Medicina de los Estados Unidos (IOM) y "An organisation with Memory $^{\text {"(2) }}$ del Ministerio de Salud del Reino Unido publicado en el año 2000. El informe "To ERR is human" concluyó que entre 44.000 a 98.000 personas mueren al año en los hospitales de Estados Unidos por EA prevenibles. Los errores médicos en Estados Unidos se situaron como la octava causa de mortalidad, por encima de la mortalidad producida por lesiones de accidente de tránsito, por cáncer de mama o SIDA. Ambos informes coincidieron que el error es algo muy habitual en la práctica asistencial, y que se da en aproximadamente un 10\% de las intervenciones.

En algunos casos el daño ocasionado es grave, incluso fatal. Se considera evento adverso cuando la circunstancia o evento no deseado ha producido daño al paciente, e incidente cuando ha habido un evento que no ha llegado a causar daño al paciente.

En España, se han realizado dos estudios iniciales para conocer los eventos adversos relacionados con la asistencia sanitaria. Primero se realizó el Estudio Nacional de Efectos Adversos Ligados a la Hospitalización, ENEAS 2005(3) , en el que se estimó que la incidencia de EA fue del 9,3\%, de los que casi el 42,8\% se podrían haber evitado. Posteriormente se elaboró el estudio APEAS(4), Estudio sobre la Seguridad de los Pacientes en la Atención Primaria de Salud (2008) en el que se observó que la frecuencia de EA es menor que en la asistencia hospitalaria y, además, predominan los EA de carácter leve. A pesar de ello, por la intensidad del uso de la atención primaria que hace la población, la seguridad del paciente es muy importante también en el primer nivel asistencial, si tenemos en cuenta que los EA pueden afectar a un $7 \%$ de ciudadanos en un año, y que el 70\% de los EA son evitables.

Existen diversos fuentes de captación de EA: indicadores de seguridad(5), la notificación voluntaria ${ }^{(6)}$, la notificación obligatoria, las quejas y reclamaciones o la revisión de historias clínicas ${ }^{(7)}$ Es frecuente el uso de plataformas que permiten a los profesionales sanitarios notificar de manera anónima y voluntaria un posible EA o una situación predisponente al error (SNEA, SiNASP) ${ }^{(8,9)}$. El sistema SNEA tiene diversas características que pueden considerarse ventajas e inconvenientes. Dentro 
de las ventajas está el que no es punitivo, que permite hacer presente el concepto de EA en una organización y que ello genera cultura de seguridad(10,11). Pero hay que considerar que el SNEA también tiene inconvenientes, la falta de cultura de notificar, el miedo a las represalias, el conocimiento poco preciso del concepto de EA y el escaso tiempo disponible para notificar, hace que las notificaciones que se reciben sean la punta del iceberg de la realidad. Por ello es necesario profundizar más en la búsqueda de casos utilizando otros sistemas de detección como puede ser el "Trigger Tool" (TT). El TT ha sido creado por el Institute for Healthcare Improvement (I.H.I.) y se basa en un cribado retrospectivo de historias clínicas para detectar parámetros o situaciones ("Triggers") que se supone que están relacionados con un $E A^{(12)}$. Es una herramienta empleada a nivel internacional en centros y sistemas sanitarios de distinta naturaleza que mide la frecuencia de aparición de EA. El uso de TT parece que identifica mayor número de eventos adversos que otros sistemas de detección ${ }^{(13)}$

La bibliografía acerca de la utilización de los modelos de gestión del riesgo y del TT a nivel ambulatorio es escasa ${ }^{(14)}$, y no se ha encontrado ningún estudio sobre utilización de TT en la asistencia sanitaria a la población trabajadora por parte de una Mutua Colaboradora con la Seguridad Social.

El objetivo de este estudio es evaluar las diferencias observadas entre la detección de (I/EA) mediante una herramienta tipo TT y las observadas en un SNEA, ambos en una Mutua Colaboradora con la Seguridad Social.

\section{Métodos}

\section{Diseño del estudio}

Estudio descriptivo transversal.

\section{Lugar, población y tiempo}

La población de estudio es la población trabajadora atendida ambulatoriamente en una Mutua Colaboradora con la Seguridad Social de Enero a Septiembre del 2016. La mutua dispone de 90 centros ambulatorios distribuidos por toda España, dos clínicas y 500 profesionales sanitarios que trabajan en el ámbito ambulatorio. La detección de casos se ha realizado en dos fases, con el objetivo de permitir la comparación entre ambas herramientas: en primer lugar se han seleccionado los 21 casos a través del sistema de notificación voluntaria (SNEA) durante el periodo de selección del estudio siendo el total de casos recibidos por el sistema de notificación voluntaria y en segundo lugar se han seleccionado aleatoriamente 20 historias clínicas por mes, 180 historias en total, donde se ha aplicado la herramienta tipo TT. El TT se ha aplicado también en los 21 recibidos por el SNEA. Se ha comprobado también que en los 180 no hubiera ninguna notificación de EA en este periodo. En total tenemos una muestra de 201 casos por ambos sistemas. 


\section{Fuentes de información}

Las fuentes de información utilizadas han sido la plataforma de notificación voluntaria por parte de los profesionales sanitarios (SNEA) y los registros de la asistencia sanitaria que recoge la historia clínica que se revisan periódicamente.

\section{Estrategia de análisis}

En primer lugar, inspirados en la herramienta original del I.H.I. "Outpatient Adverse Event Trigger Tool" (OAETT) ${ }^{(15)}$ se diseñaron los triggers adaptados a la realidad de una Mutua Colaboradora con la Seguridad Social. El resultado final han sido los siguientes 11 Triggers: diagnóstico complejo, derivaciones de admisión o alta a distintos niveles asistenciales o especialistas, más de 5 visitas, procedimiento quirúrgico, polimedicado, quejas y reclamaciones, utilización de antibiótico, cambio o suspensión abrupta de la medicación, visita post alta laboral, baja laboral posterior a primera visita médica, cambio de contingencia (ver Tabla 1).

Seguidamente se han revisado las 201 historias clínicas buscando la existencia de triggers. Un trigger se define como un ítem concreto y fácilmente identificable en la historia de un paciente que representa una oportunidad (o pista) que puede llevarnos a un Incidente o a un Evento Adverso. Cuando se han detectado triggers positivos se ha procedido a la revisión exhaustiva de los registros clínicos para determinar si existe algún incidente o evento adverso. En el caso de encontrar más de un incidente o EA en la misma historia clínica, se ha considerado el de mayor gravedad.

\section{Variables en estudio}

\section{Variable dependiente}

La variable dependiente recoge la presencia o no de I/EA en forma binaria (si hay I/EA ó No hay I/EA). También se ha considerado la segmentación del I/EA según tipología, severidad y evitabilidad( ${ }^{(16)}$. En relación al tipo de I/EA se ha clasificado en: equipos/dispositivos, procedimientos quirúrgicos/pruebas diagnósticas/procedimientos terapéuticos, infraestructura/edificios/enseres, medicación, gestión organizativa/recursos, caídas/accidentes pacientes, infecciones relacionadas con la asistencia (ira), procesos administrativos, identificación. Para la severidad se ha utilizado la clasificación "The National Coordinating Council For Medication Error Reporting and Prevention (NCC MERP) Index for categorizing errors"(15). Esta clasificación es la misma que se utiliza en el OAETT (Outpatient Adverse Event Trigger Tool elaborados por IHI y Kaiser Permanente) y que además permite diferenciar entre incidente y evento adverso. Para la evitabilidad se ha utilizado una clasificación con 6 categorías ordenadas, donde 1 sería que no hay evidencia que sea evitable y 6 que hay evidencia cierta que es evitable. 
Tabla 1. Adaptación del Outpatient Adverse Event Trigger Tool (OAETT) a un Trigger Tool ambulatorio para una Mutua Colaboradora con la Seguridad Social (MCSS)

\begin{tabular}{|c|c|c|c|}
\hline & $\begin{array}{l}\text { Outpatient } \\
\text { Adverse Event } \\
\text { Trigger Tool }\end{array}$ & $\begin{array}{l}\text { Trigger Tool } \\
\text { ambulatorio para } \\
\text { MCSS }\end{array}$ & Descripción de la adaptación \\
\hline Trigger 1 & $\begin{array}{l}\text { Nuevo } \\
\text { Diagnóstico de } \\
\text { cáncer }\end{array}$ & $\begin{array}{l}\text { Diagnóstico } \\
\text { complejo }\end{array}$ & $\begin{array}{l}\text { Nuestro ámbito es fundamentalmente } \\
\text { traumatológico. Se han considerado } \\
\text { diagnósticos complejos que necesitarán } \\
\text { procedimientos quirúrgicos, pruebas } \\
\text { diagnósticas, procedimientos terapéuticos o } \\
\text { derivaciones entre niveles asistenciales }\end{array}$ \\
\hline Trigger 2 & $\begin{array}{l}\text { Visita de } \\
\text { Enfermería a } \\
\text { domicilio }\end{array}$ & $\begin{array}{l}\text { Derivaciones de } \\
\text { admisión o alta a } \\
\text { distintos niveles } \\
\text { asistenciales/ } \\
\text { especialistas }\end{array}$ & $\begin{array}{l}\text { No se dispone de servicio de atención } \\
\text { domiciliaria. Se han considerado derivaciones } \\
\text { de admisión o alta a distintos niveles } \\
\text { asistenciales y la derivación a especialistas, } \\
\text { entendiendo que puede haber problemas de } \\
\text { continuidad asistencial }\end{array}$ \\
\hline Trigger 3 & Alta hospitalaria & Más de 5 visitas & $\begin{array}{l}\text { Se considera más de } 5 \text { visitas realizadas, } \\
\text { entendiendo que un proceso con más visitas } \\
\text { tiene más contactos de atención sanitaria y es } \\
\text { más susceptible a errores }\end{array}$ \\
\hline Trigger 4 & $\begin{array}{l}\text { Más de dos } \\
\text { especialistas }\end{array}$ & $\begin{array}{l}\text { Procedimiento } \\
\text { quirúrgico }\end{array}$ & $\begin{array}{l}\text { No es habitual que actúen dos especialistas. } \\
\text { Se ha adaptado por el trigger de procedimiento } \\
\text { quirúrgico, pues cualquier procedimiento } \\
\text { que implique cirugía puede ocasionar EA } \\
\text { (infecciones relacionadas con la asistencia } \\
\text { sanitaria, dehiscencia de heridas, etc.). Es el } \\
\text { trigger } 5 \text { del OAETT }\end{array}$ \\
\hline Trigger 5 & $\begin{array}{l}\text { Procedimiento } \\
\text { quirúrgico }\end{array}$ & $\begin{array}{l}\text { Más de } 5 \\
\text { medicamentos }\end{array}$ & $\begin{array}{l}\text { Corresponde al } 7 \text { del OAETT. Será } \\
\text { positivo cuando se administren más de } 5 \\
\text { medicamentos }\end{array}$ \\
\hline Trigger 6 & $\begin{array}{l}\text { Visita a } \\
\text { Urgencias }\end{array}$ & $\begin{array}{l}\text { Quejas y } \\
\text { reclamaciones }\end{array}$ & $\begin{array}{l}\text { Se buscan quejas y reclamaciones que consten } \\
\text { en la Historia Clínica. Asimilable al trigger } 9 \\
\text { OAETT }\end{array}$ \\
\hline Trigger 7 & $\begin{array}{l}\text { Más } 5 \\
\text { medicamentos }\end{array}$ & $\begin{array}{l}\text { Utilización de } \\
\text { antibiótico }\end{array}$ & $\begin{array}{l}\text { Fundamentalmente se presentan patologias } \\
\text { musculoesqueléticas que raramente precisan } \\
\text { antibióticos a no ser que haya alguna infección } \\
\text { de partes blandas o de alguna articulación }\end{array}$ \\
\hline Trigger 8 & $\begin{array}{l}\text { Cambio de } \\
\text { médico }\end{array}$ & $\begin{array}{l}\text { Cambios/ } \\
\text { suspensión } \\
\text { tratamiento }\end{array}$ & $\begin{array}{l}\text { La mayoría de casos son de corta duración } \\
\text { y es infrecuente un cambio de médico. Se ha } \\
\text { considerado en su lugar un cambio abrupto o } \\
\text { una suspensión de la medicación posiblemente } \\
\text { hayan sido debido a un EA }\end{array}$ \\
\hline Trigger 9 & $\begin{array}{l}\text { Carta de } \\
\text { reclamación }\end{array}$ & $\begin{array}{l}\text { Visita tras Alta } \\
\text { laboral }\end{array}$ & $\begin{array}{l}\text { Si tras la emisión de un alta laboral el paciente } \\
\text { vuelve a consulta se puede haber generado un } \\
\text { EA }\end{array}$ \\
\hline Trigger 10 & $\begin{array}{l}\text { Más de } 3 \\
\text { llamadas } \\
\text { enfermería/ } \\
\text { semana }\end{array}$ & $\begin{array}{l}\text { Baja laboral tras } \\
1^{\text {a }} \text { visita }\end{array}$ & $\begin{array}{l}\text { Cuando una baja laboral se da posteriormente } \\
\text { a una primera visita hay que cerciorarse que no } \\
\text { sea debido a un EA }\end{array}$ \\
\hline Trigger 11 & $\begin{array}{l}\text { Valor anormal } \\
\text { de laboratorio }\end{array}$ & Contingencia & $\begin{array}{l}\text { Fundamentalmente se presentan patologias } \\
\text { musculoesqueléticas que raramente precisan } \\
\text { analíticas. La valoración de la contingencia y el } \\
\text { hecho que en función de la misma se trate en } \\
\text { entes diferentes puede ocasionar duplicidad de } \\
\text { pruebas, retrasos diagnósticos o de tratamiento }\end{array}$ \\
\hline
\end{tabular}




\section{Variables explicativas}

La variable explicativa principal es el tipo de sistema utilizado para detectar los I/EA, con valores SNEA (incluidas las quejas) y TT. También se han considerado las variables sexo, la edad según cuartiles ( $\leq 33,34-41,42-49$ y $\geq 50$ años), sector (primario, secundario y terciario) y duración de la incapacidad temporal (IT) en cuartiles $(\leq 14,15-28,29-53$ y $\geq 54$ días $)$.

\section{Análisis estadístico}

Se ha realizado una descripción de los 201 casos analizados según sexo, edad, sector y duración de la IT. A continuación se ha calculado la proporción de I/EA para el SNEA y el TT según sexo, edad, sector y duración de la IT. La prueba estadística utilizada para valorar las diferencias entre proporciones ha sido la prueba exacta de Fisher. Seguidamente, se ha obtenido la proporción de I/EA para el SNEA y el TT según tipología, severidad y evitabilidad. Finalmente, se ha evaluado la concordancia entre el sistema SNEA y el TT utilizando la proporción de concordancia positiva (I/EA), proporción de concordancia negativa (no I/EA) e índice Kappa. Para conocer más detalles sobre la definición y utilidad de acompañar el índice Kappa de las proporciones de concordancia positiva y negativa, se puede consultar el trabajo de Henrica C W et al(17). Los programas utilizados han sido Excel 2010 y Stata $9^{(18)}$.

\section{Aspectos éticos}

Los datos han sido tratados de forma agregada y anónima, de tal manera que no permiten la identificación, de acuerdo al cumplimiento del Reglamento del Parlamento Europeo y del Consejo (UE) 2016/679 y el Reglamento General de Protección de Datos (RGPD).

\section{Resultados}

La muestra analizada presentó una proporción mayor de hombres $(66,2 \%)$ y población trabajadora del sector terciario (57,8\%), y secundario (37,7\%). El 75\% de la muestra tiene más de 49 años y presenta una duración de la IT superior a 53 días (Tabla 2).

Mediante el sistema SNEA se detectaron 21 I/EA (15 Incidentes y 6 Eventos Adversos). Con la herramienta TT se detectaron 83 I/EA (23 incidentes y 60 Eventos Adversos). Al comparar la proporción de detección de casos de ambos sistemas es importante destacar la existencia de diferencias importantes, específicamente así el TT detecta casos en un $41,3 \%$ de las revisiones mientras que para el Sistema SNEA es de 10,3\% en la muestra de historias configurada, siendo estas diferencias también estadísticamente significativas $(p<0,001)$. En los valores de cada variable dentro del grupo de SNEA y TT, solo la variable duración presenta diferencias estadísticamente significativas. En el cuartil con una duración superior a 54 días se 
encuentra una proporción del $20 \%$ de eventos adversos y que es con diferencia el cuartil donde más eventos adversos se detectan en el sistema SNEA $(p<0,001)$. En el sistema TT se observó que en los dos cuartiles superiores es donde se encuentra mayor proporción de eventos adversos con un 48,9 y $51,9 \%$, respectivamente $(p=0,017)$.

Tabla 2. Descripción de la muestra (frecuencia y porcentaje). Enero-Septiembre 2016.

\begin{tabular}{|c|c|c|}
\hline & $\mathrm{n}$ & $(\%)$ \\
\hline \multicolumn{3}{|l|}{ Sexo } \\
\hline Hombre & 133 & $(66,2)$ \\
\hline Mujer & 68 & $(33,8)$ \\
\hline \multicolumn{3}{|l|}{ Edad (años) } \\
\hline$<34$ & 52 & $(25,9)$ \\
\hline $34-41$ & 54 & $(26,9)$ \\
\hline $42-49$ & 45 & $(22,4)$ \\
\hline$\geq 50$ & 50 & $(24,9)$ \\
\hline \multicolumn{3}{|l|}{ Sector } \\
\hline Primario & 9 & $(4,5)$ \\
\hline Secundario & 75 & $(37,7)$ \\
\hline Terciario & 115 & $(57,8)$ \\
\hline \multicolumn{3}{|l|}{ Duración (días) } \\
\hline$<15$ & 48 & $(25,5)$ \\
\hline $15-28$ & 47 & $(25,0)$ \\
\hline $29-53$ & 46 & $(24,5)$ \\
\hline$\geq 54$ & 47 & $(25,0)$ \\
\hline Total & 201 & (100) \\
\hline
\end{tabular}

a. 2 valores perdidos. c. 13 valores perdidos.

b. Sector. primario: agricultura, ganadería, pesca, silvicultura y minería; Sector secundario: industria,

construcción y producción de energía eléctrica; Sector terciario: comercio, transporte, turismo, educación,

sanidad y administración.

c. Duración. días de incapacidad temporal.

El tipo de I/EA más frecuente en ambos sistemas de detección fue el de procedimientos quirúrgicos/pruebas diagnósticas/procedimientos terapéuticos, que se observa en un $5,5 \%$ de los SNEA y en un $20,4 \%$ de los casos revisados mediante TT. En el TT los procesos administrativos (10\%) y la medicación $(6,5 \%)$ fueron los siguientes en frecuencia. Por severidad, en el SNEA detecta más incidentes (niveles A-D) que EA (niveles E-I) (15 frente a 6) siendo las circunstancias con capacidad de generar error (A) la severidad más habitual en los casos detectados en el SNEA. El grado más frecuente en el TT es el daño temporal al paciente que requiere intervención (E) con un 23,9\%. Respecto a la evitabilidad, destaca que el 14,9\% de pacientes que presentan un EA con evidencia sólida de poder ser evitados. 
Tabla 3. Frecuencia y proporción de Incidente/Evento Adverso, según la clasificación del Sistema de Notificación de Eventos Adversos (SNEA) y del Trigger Tool (TT) (n=201). Enero-Septiembre 2016.

\begin{tabular}{|c|c|c|c|c|c|c|c|}
\hline & \multicolumn{2}{|c|}{ SNEA } & \multirow[b]{2}{*}{$\mathrm{p}_{1}$} & \multicolumn{2}{|c|}{ TT } & \multirow[b]{2}{*}{$\mathrm{p}_{1}$} & \multirow[b]{2}{*}{$\mathrm{p}_{2}$} \\
\hline & c & $\operatorname{Pr}(\%)$ & & c & $\operatorname{Pr}(\%)$ & & \\
\hline \multicolumn{8}{|l|}{ Sexo } \\
\hline Hombre & 12 & $(9,0)$ & 0,465 & 54 & $(40,6)$ & 0,880 & $<0,001$ \\
\hline Mujer & 9 & $(13,2)$ & & 29 & $(42,7)$ & & $<0,001$ \\
\hline \multicolumn{8}{|l|}{ Edad } \\
\hline$<34$ & 4 & $(7,7)$ & 0,220 & 18 & $(34,6)$ & 0,575 & 0,001 \\
\hline $34-41$ & 9 & $(16,7)$ & & 22 & $(40,7)$ & & 0,010 \\
\hline $42-49$ & 2 & $(4,4)$ & & 22 & $(48,9)$ & & $<0,001$ \\
\hline$\geq 50$ & 6 & $(12,0)$ & & 21 & $(42,0)$ & & 0,001 \\
\hline \multicolumn{8}{|l|}{ Sector } \\
\hline Primario & 1 & $(11,1)$ & 0,589 & 3 & $(33,3)$ & 0,193 & 0,576 \\
\hline Secundario & 9 & $(12,0)$ & & 37 & $(49,3)$ & & $<0,001$ \\
\hline Terciario & 10 & $(8,7)$ & & 42 & $(36,5)$ & & $<0,001$ \\
\hline \multicolumn{8}{|l|}{$\begin{array}{r}\text { Duración } \\
\text { (días) }\end{array}$} \\
\hline$<15$ & 1 & $(2,1)$ & $<0,001$ & 12 & $(25,0)$ & 0,017 & 0,002 \\
\hline $15-28$ & 1 & $(2,2)$ & & 15 & $(31,9)$ & & $<0,001$ \\
\hline $29-53$ & 0 & $(0,0)$ & & 22 & $(47,8)$ & & $<0,001$ \\
\hline$\geq 54$ & 11 & $(23,4)$ & & 28 & $(59,6)$ & & 0,001 \\
\hline Total & 21 & $(10,5)$ & & 83 & $(41,3)$ & & $<0,001$ \\
\hline
\end{tabular}

c: número de I/EA

$\operatorname{Pr}(\%)$ : Número de I/EA por 100 pacientes

p1: valor p para comparar la proporción de I/EA en los valores de cada variable dentro del grupo de SNEA Y TT respectivamente

p2: valor p para comparar la proporción de I/EA en el grupo de SNEA frente a TT.

El índice Kappa mostró un valor de concordancia baja (Kappa=0,12) (Tabla 5) lo que denota la pequeña coincidencia de I/EA detectados por ambos sistemas. La proporción de concordancia negativa fue mayor que la de concordancia positiva (74,5\% frente a un $26,9 \%)$. 
Tabla 4. Número y proporción de casos Incidentes/Eventos Adversos según tipología, severidad y evitabilidad para Sistema de Notificación de Eventos Adversos (SNEA) y Trigger Tool (TT). Enero-Septiembre 2016.

\begin{tabular}{|c|c|c|c|c|}
\hline & \multicolumn{2}{|c|}{ SNEA } & \multicolumn{2}{|c|}{ TT } \\
\hline & c & $\operatorname{Pr}(\%)$ & c & $\operatorname{Pr}(\%)$ \\
\hline \multicolumn{5}{|l|}{ Tipología } \\
\hline Equipos/Dispositivos & 1 & $(0,5)$ & 0 & $(0,0)$ \\
\hline Proc. Quirúrgicos/Pruebas DX/Proc. Terapéuticos & 11 & $(5,5)$ & 41 & $(20,4)$ \\
\hline Infraestructura/Edificios/Enseres & 0 & $(0,0)$ & 0 & $(0,0)$ \\
\hline Medicación & 1 & $(0,5)$ & 13 & $(6,5)$ \\
\hline Gestión Organizativa/Recursos & 2 & $(1,0)$ & 3 & $(1,5)$ \\
\hline Caídas/Accidentes pacientes & 3 & $(1,5)$ & 2 & $(1,0)$ \\
\hline IRA (Infecciones Relacionadas Asistencia) & 1 & $(0,5)$ & 4 & $(2,0)$ \\
\hline Procesos Administrativos & 1 & $(0,5)$ & 20 & $(10,0)$ \\
\hline Identificación & 1 & $(0,5)$ & 0 & $(0,0)$ \\
\hline \multicolumn{5}{|l|}{ Severidad } \\
\hline Circunstancias con capacidad de causar error (A) & 11 & $(5,5)$ & 2 & $(1,0)$ \\
\hline Error que no llega al paciente (B) & 2 & $(1,0)$ & 7 & $(3,5)$ \\
\hline Error que llega al paciente pero no causa daño ( $C$ ) & 2 & $(1,0)$ & 14 & $(7,0)$ \\
\hline $\begin{array}{l}\text { Error que llega al paciente y necesita } \\
\text { monitorización para saber que no hay daño (D) }\end{array}$ & 0 & $(0,0)$ & 4 & $(2,0)$ \\
\hline $\begin{array}{l}\text { Daño temporal al paciente que requiere } \\
\text { intervención ( } E \text { ) }\end{array}$ & 4 & $(2,0)$ & 48 & $(23,9)$ \\
\hline $\begin{array}{l}\text { Daño temporal al paciente que requiere } \\
\text { hospitalización (F) }\end{array}$ & 0 & $(0,0)$ & 7 & $(3,5)$ \\
\hline Daño permanente (G) & 2 & $(1,0)$ & 1 & $(0,5)$ \\
\hline Intervención para mantener la vida (H) & 0 & & 0 & \\
\hline Paciente muerto (I) & 0 & $(0,0)$ & 0 & $(0,0)$ \\
\hline \multicolumn{5}{|l|}{ Evitabilidad } \\
\hline Poca o nula evidencia (1) & & & 9 & $(4,5)$ \\
\hline De leve a moderada evidencia (2) & - & - & 13 & $(6,5)$ \\
\hline Menos del $50 \%$ de evidencia (3) & - & & 9 & $(4,5)$ \\
\hline Más del 50\% de evidencia (4) & - & & 19 & $(9,5)$ \\
\hline Evidencia sólida (5) & - & & 30 & $(14,9)$ \\
\hline Evidencia cierta (6) & - & & 3 & $(1,5)$ \\
\hline
\end{tabular}

c: Número de I/EA (incidentes y eventos adversos).

$\operatorname{Pr}(\%)$ : Número de I/EA por 100 pacientes. 
Tabla 5. Concordancia entre la clasificación de I/EA del SNEA y del TT. Enero-Septiembre 2016.

\begin{tabular}{|c|c|c|c|c|c|}
\hline $\mathrm{P}_{\mathrm{C}+}$ & (IC 95\%) & $\mathrm{P}_{\mathrm{C}-}$ & (IC 95\%) & $\mathrm{K}$ & IC $95 \%$ \\
\hline 26,9 & $(18,7-36,5)$ & 74,5 & $(69,2-79,3)$ & 0,12 & $(0,03-0,22)^{\star}$ \\
\hline
\end{tabular}

\section{Discusión}

Los resultados muestran que el TT detecta en nuestro estudio 4 veces más sucesos adversos que el sistema SNEA. Se observa una baja concordancia, lo que indica la complementariedad de ambos sistemas, siendo recomendable una aplicación conjunta. Específicamente, el sistema SNEA detecta más incidentes y en especial situaciones capaces de generar EA. El sistema TT detecta más EA.

Una herramienta tipo Trigger Tool puede adaptarse a cualquier realidad, con la cautela de definirlos de manera específica en función del tipo de organización o servicio que se está considerando. Este trabajo ha utilizado como punto de partida el TT basado en la herramienta OAETT. Este se ha adaptado teniendo en cuenta la realidad de una Mutua Colaboradora con la Seguridad Social, en la que no solo se presta atención sanitaria sino que también se gestionan prestaciones económicas. En este contexto se han incorporado triggers que no están presentes en la herramienta original pero son necesarios para este tipo de organización. Se trata de triggers de medicina evaluadora y están relacionados con los actos de emitir una baja o un alta, pues a pesar de tener componentes administrativos pueden tener impacto en el proceso asistencial del paciente. Los triggers también están diseñados en relación a las particularidades de los procesos asistenciales de las mutuas que son de corta duración y mayoritariamente musculoesquéticos. A la hora de interpretar los resultados se debe tener en cuenta los TT finalmente considerados. Esto podría explicar la mayor proporción de I/EA detectados en comparación con el sistema SNEA. Otra consideración es que se ha incluido la existencia o bien de un incidente o bien de un evento adverso. En el diseño inicial del IHI la estimación de eventos adversos mediante herramientas Trigger Tool, no incluía los incidentes, pero en nuestro caso nos ha parecido importante incluirlos, porque permiten elaborar iniciativas y planes de seguridad con mayor conocimiento y extensión. También se debe tener en cuenta que en el caso de que se haya encontrado más de un I/EA en una historia clínica, solo se ha contabilizado uno. También hemos iniciado el estudio de sensibilidad, especificidad y valores predictivos de cada uno de los TT para mejorar la adaptación del instrumento TT a la realidad de una mutua laboral.

El concepto de complementariedad que se ha encontrado entre ambos sistemas, también se apunta en un estudio en relación al uso de diferentes sistemas de notificación de eventos adversos ${ }^{(19)}$, que concluye que la inclusión de varios sistemas de notificación incrementa el número de eventos adversos detectados. 
El uso de una herramienta TT se basa en una búsqueda activa, por tanto no hay que esperar que los profesionales efectivamente notifiquen un I/EA como ocurre en un sistema de notificación voluntaria. También permite una buena estimación de EA y por tanto presenta un escenario más parecido a la realidad. Este punto es especialmente importante pues permite la implementación de iniciativas de seguridad acordes con las necesidades reales. Es un sistema de detección centrado en el daño, no en el error. Este sistema analiza el daño causado y lo orienta al sistema lo que es esencial en la concepción moderna de seguridad del paciente. Otra ventaja es que es de aplicación relativamente simple y barata relacionada con la auditoria de historias clínicas que ya se realiza en nuestra institución Sin embargo, también presenta inconvenientes. Los revisores necesitan entrenamiento y formación en el concepto de incidente, EA y en la propia herramienta TT. Hay que estar familiarizado con el diseño de los triggers diseñados, así como en la evaluación de la severidad y la evitabilidad cuando se detecta un I/EA. Aunque no es imprescindible, el contar con más de un revisor puede dar mayor solidez a la consistencia de los datos obtenidos. En nuestro sistema se realiza una doble lectura sistemática en una muestra de las historias revisadas.Como el TT se basa en la revisión de la información contenida en la Historia Clínica, puede que no se detecten algunos I/ EA por falta de registro en la misma. Por ejemplo, una caída en la sala de espera difícilmente se anotará en la HC y por tanto no será detectado por el TT.

A diferencia de la utilización de la herramienta TT con las recomendaciones del IHI, en este estudio aparte de incluir incidentes, también se ha incluido la evitabilidad y el tipo de incidente/EA. A tenor de los resultados del estudio, estas tres variables aportan información relevante y útil para determinar un plan de seguridad. Entendemos que no se debería prescindir de los incidentes en una revisión sistemática de Historias Clínicas.

En resumen, el Trigger Tool se muestra como una buena herramienta para la detección de I/EA que puede complementar la obtenida mediante el SNEA en la realidad de una Mutua Colaboradora con la Seguridad Social. En este estudio se realiza un primer abordaje de la adaptación del instrumento a la realidad de una mutua laboral, siendo necesario el profundizar en la sensibilidad, especificidad y valores predictivos de cada uno de los TT para mejorar la adaptación. En este contexto se recomienda que para desarrollar e implementar iniciativas y planes de seguridad del paciente, el TT debiera incluir incidentes, tipo de I/EA y evitabilidad.

\section{Bibliografía}

1. Kohn LT, Corrigan JM, Donaldson MS, eds. (Committee on Quality of Health Care in America, Institute of Medicine). To Err is Human: Building a Safer Health System. Washington, DC, USA: National Academies Press; 2000.

2. Donaldson L. An Organisation With a Memory. Clin Med (Lond). 2002;2(5):452-7. doi:10.7861/clinmedicine.2-5-452 
3. Ministerio de Sanidad y Consumo. Estudio Nacional sobre los Efectos Adversos Ligados a la Hospitalización. ENEAS 2005. Madrid: Ministerio de Sanidad y Consumo. 2006:170. doi:351-06-009-2

4. Ministerio de Sanidad y Consumo. Estudio APEAS. Estudio sobre La Seguridad de los Pacientes en Atención Primaria de Salud. Madrid: Ministerio de Sanidad y Consumo. 2008:192.

5. Agency for Healthcare Research and Quality. Guide to Patient Safety Indicators. Health Care (Don Mills). 2004. [citado 14 Abr 2020]. (Disponible en http://www. qualityindicators.ahrq.gov).

6. Leape LL.Reporting of Adverse Events. N Engl J Med. 2002;347(20):1633-8.

7. Brennan TA, Leape LL, Laird NM, Hebert L, Localio AR, Lawthers AG, et al. Incidence of adverse events and negligence in hospitalized patients: results of the Harvard Medical Practice Study I. 2004;(2):145-51.

8. Bañeres J, Orrego C, Suñol R, Ureña V. Los sistemas de registro y notificación de efectos adversos y de incidentes: una estrategia para aprender de los errores. Rev Calid Asist. 2005;20(4):216-222. doi:10.1016/S1134-282X(08)74754-7.

9. Oliva G, Álava F, Navarro L, Esquerra M, Lushchenkova O, Davins J, et al. Notificación de incidentes relacionados con la seguridad del paciente en los hospitales de Cataluña durante el período 2010-2013. Med Clin (Barc). 2014;143(SUPPL. 1):5561. doi:10.1016/j.medcli.2014.07.012

10. Manzanera R, Mira JJ, Plana M, Moya D, Guilabert M, Ortner J. Patient Safety Culture in Mutual Insurance Companies in Spain. J Patient Saf. 2017. doi:10.1097/ PTS.0000000000000300. [Epub ahead of print]

11. Manzanera R, Plana M, Moya D, Ortner J, Mira JJ. Implantación de buenas prácticas en calidad y seguridad. Estudio descriptivo en una mutua laboral. Rev Calid Asist. 2016;31(3):168-72. doi:10.1016/j.cali.2015.10.002

12. Griffin FA, Resar RK. IHI Global Trigger Tool for Measuring Adverse Events (Second Edition). IHI Innovation Series white paper. Cambridge, MA: Institute for Healthcare Improvement; 2009. [citado 14 Abr 2020]. (Disponible en www.IHI.org)

13. Classen DC, Resar R, Griffin F, Federico F, Frankel T, Kimmel N, et al. "Global Trigger Tool" Shows that Adverse Events in Hospitals maybe ten times greater than previously measured. Health Aff (Millwood). 2011;30(4):581-9. doi:10.1377/ hlthaff.2011.0190.

14. Grau N, Manzanera R, Orrego C, Ortner J, Vives A, Saurí C, et al. Risk Management in the Ambulatory Care Process in a Mutual Benefit Association Covering Work-Related Accidents and Diseases. J Patient Saf. 2018. doi:10.1097/ PTS.0000000000000542.

15. Institute for Healthcare Improvement. IHI Outpatient Adverse Event Trigger Tool. 2006. [Citado 14 Abr 2020]. (Disponible en http://www.ihi.org/resources/Pages/Tools/OutpatientAdverseEventTriggerTool.aspx). 
16. Hibbert PD, Molloy CJ, Hooper TD, Wiles LK, Runciman WB, Lachman P, et al. The application of the Global Trigger Tool: a systematic review. Int J Qual Heal Care. 2016;28(6):640-649. doi:https://doi.org/10.1093/intqhc/mzw115

17. de Vet HC, Mokkink LB, Terwee CB, Hoekstra OS, Knol DL. Clinicians are right not to like Cohen's Kappa. BMJ. 2013;346:f2125. doi:10.1136/bmj.f2125.

18. StataCorp. 2005. Stata Statistical Software: Release 9. College Station, TX: StataCorp LP.

19. Menéndez MD, Rancaño I, García V, Vallina $C$, Herranz V, Vázquez F. Uso de diferentes sistemas de notificación de eventos adversos: ¿mucho ruido y pocas nueces? Rev Calid Asist. 2010;25(4):232-236. doi:10.1016/j.cali.2010.02.001 